\title{
Baicalin, baicalein and wogonin inhibits high glucose-induced vascular inflammation in vitro and in vivo
}

\author{
Sae-Kwang $\mathrm{Ku}^{1} \mathcal{E}$ Jong-Sup Bae $\mathrm{e}^{2, *}$ \\ ${ }^{1}$ Department of Anatomy and Histology, College of Korean Medicine, Daegu Haany University, Gyeongsan 38610, ${ }^{2}$ College of Pharmacy, \\ CMRI, Research Institute of Pharmaceutical Sciences, Kyungpook National University, Daegu 41566, Korea
}

\begin{abstract}
Vascular inflammatory process has been suggested to play a key role in initiation and progression of atherosclerosis, a major complication of diabetes mellitus. Thus, in this study, we attempted to determine whether three structurally related polyphenols found in the Chinese herb Huang Qui, namely baicalin, baicalein, and wogonin, can suppress vascular inflammatory processes induced by high glucose $(\mathrm{HG})$ in human umbilical vein endothelial cells (HUVECs) and mice. Data showed that HG induced markedly increased vascular permeability, monocyte adhesion, expressions of cell adhesion molecules (CAMs), formation of reactive oxygen species (ROS) and activation of nuclear factor (NF)-kB. Remarkably, all of the above mentioned vascular inflammatory effects of $\mathrm{HG}$ were attenuated by pretreatment with baicalin, baicalein, and wogonin. Vascular inflammatory responses induced by HG are critical events underlying development of various diabetic complications, therefore, our results suggest that baicalin, baicalein, and wogonin may have significant therapeutic benefits against diabetic complications and atherosclerosis. [BMB Reports 2015; 48(9): 519-524]
\end{abstract}

\section{INTRODUCTION}

Diabetes mellitus (DM) is one of the metabolic disorders associated with various diseases, including arteriosclerosis, nephritis, and hypertension $(1,2)$. The most common type of DM is Non-insulin dependent diabetes mellitus (NIDDM) $(1,2)$; it is widespread, a growing threat to global public health, and has the biggest impact on adults of working age in developing countries (3). Often DM is not recorded as the cause of death, but globally it was considered the fifth leading cause of death

${ }^{*}$ Corresponding author. Tel: +82-53-950-8570; Fax: +82-53-9508557; E-mail: baejs@knu.ac.kr

http://dx.doi.org/10.5483/BMBRep.2015.48.9.017

Received 28 January 2015, Revised 17 February 2015, Accepted 2 March 2015

Keywords: Baicalein, Baicalin, Diabetes mellitus; Wogonin, High glucose in 2000, after communicable diseases, cardiovascular disease, cancer, and injuries (4). In 2011, there were 366 million people with DM in the world, and this number is expected to rise to 552 million by 2030; approximately $80 \%$ of these people reside in developing countries (1). Extensive investigations into the pathogenesis of NIDDM have identified two endocrine defects functionally: insulin deficiency and insulin resistance $(1,2)$. In modern medicine, an effective cure for DM is unavailable (5). Thus, the research for more effective agents than others has continued. Many indigenous medicinal plants are useful in controlling DM successfully; some effective compounds were evaluated and found (6). The World Health Organization has recommended the analysis of the potential of plants as effective therapeutic agents (7).

Scutellaria baicalensis Georgi (Huang-qin) is a medicinal herb used to treat various types of inflammatory diseases, hepatitis, tumors, and diarrhea in East Asian countries such as China, Korea, Taiwan, and Japan (8). This plant contains many bioactive compounds including phenethyl alcohols, sterols, essential oils, and amino acids. Baicalin, baicalein, or wogonin is a single flavonoid isolated from Scutellaria baicalensis Georgi (9). The inhibitory activities of these flavonoids on vascular inflammation have been attributed to suppress LPS-induced pro-inflammatory responses, for example, the productions of nitric oxide and tumor necrosis factor- $\alpha$, and nitric oxide synthase gene expressions $(10,11)$. However, the effects of baicalin, baicalein, or wogonin on high-glucose (HG)-induced inflammatory responses have not been reported. Therefore, in the current study, we attempted to determine whether baicalin these compounds can suppress the vascular inflammatory responses induced by $\mathrm{HG}$ in human endothelial cells and in mice.

\section{RESULTS AND DISCUSSION}

Baicalin, baicalein, and wogonin are three structurally similar polyphenols, which are active compounds found in the Chinese herb Huang-qin. In this study, the effects of these polyphenols on HG-induced vascular inflammation were determined in vitro and in vivo.

ISSN: 1976-670X (electronic edition)

Copyright (C) 2015 by the The Korean Society for Biochemistry and Molecular Biology

(c) This is an open-access article distributed under the terms of the Creative Commons Attribution Non-Commercial License (http://creativecommons.org/licenses/by-nc/4.0) which permits unrestricted non-commercial use, distribution, and reproduction in any medium, provided the original work is properly cited. 
Effects of polyphenols on HG-induced disruption of the endothelial barrier function of Human Umbilical Vein Endothelial Cells (HUVECs) and in mice

Previous evidence has suggested that endothelial dysfunction and damage are precursors to vascular complications in DM (12). Hyperglycemia is the main precursor to all types of diabetic microvascular disease and may be involved in the pathogenesis of macrovascular complications as well (12-14). In addition, endothelial cell permeability is impaired, and may be increased, by the high concentrations of extracellular glucose in diabetes mellitus (15). Leakage of serum proteins, particularly albumin, through the endothelium is observed in the retinal vessels of early-stage diabetes mellitus $(15,16)$. Increased endothelial cell permeability in the larger vessels leads to the development of interstitial edema and may result in the enhancement of cell proliferation and matrix production (14). Therefore, we first investigated the effects of glucose on the albumin permeability of endothelial cells, as shown in Fig. 1A. Treatment with HG (25 and $50 \mathrm{mM}$ ) led to a rapid increase in endothelial cell permeability (Fig. 1A). This effect began $12 \mathrm{~h}$ after incubation and reached its maximum at $24 \mathrm{~h}$ (Fig. 1B). A significant increase was observed at a glucose concentration of
$10 \mathrm{mM}$. Concentrations above $50 \mathrm{mM}$ did not increase the HG-induced permeability further (data not shown). L-glucose and D-Mannose (25 mM), which were used as an osmotic control, had no significant effect on endothelial cell permeability (Fig. 1A).

Next, we attempted to determine whether baicalin, baicalein, and/or wogonin could alter HG-induced hyperpermeability. Treatment with $10 \mu \mathrm{M}$ of each compound alone did not result in alteration of barrier integrity (Fig. 1C). As shown in Fig. 1C, treatment with baicalin, baicalein, or wogonin resulted in a dose-dependent decrease in HG-mediated membrane disruption; the minimum effect of baicalin, baicalein, or wogonin was observed at $5 \mu \mathrm{M}$. To confirm this vascular barrier protective effect in vivo, HG-mediated vascular permeability in mice was assessed. As shown in Fig. 1D, treatment with baicalin, baicalein, or wogonin resulted in significant inhibition of peritoneal leakage induced by HG. Because the average weight of a mouse is $20 \mathrm{~g}$ and the average blood volume is 2 $\mathrm{mL}$, the injected baicalin $(1.1,2.7$, or $5.4 \mu \mathrm{g} /$ mouse), baicalein $(1.8,4.5$, or $8.9 \mu \mathrm{g} / \mathrm{mouse})$, or wogonin $(1.1,2.8$, or 5.7 $\mu \mathrm{g} /$ mouse) produced concentration maximums of 2,5 , or 10 $\mu \mathrm{M}$ in the peripheral blood. To test the effects of the cellular
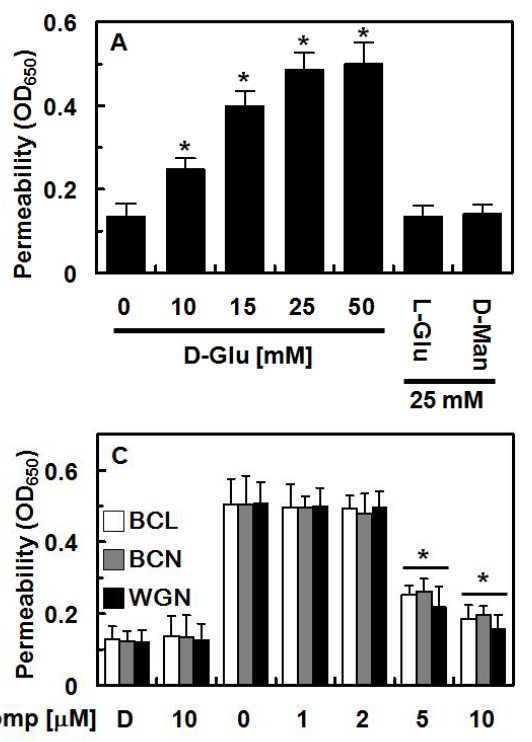
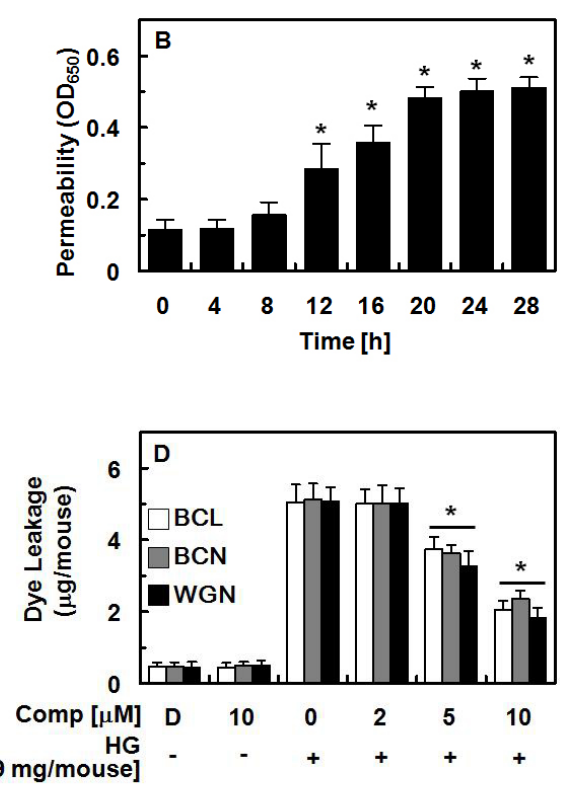
[9 mg/mouse]

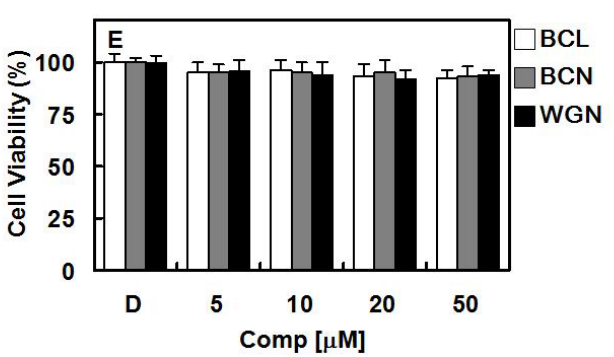

Fig. 1. Effects of polyphenols on HGmediated permeability in vitro and in vivo. (A) HUVECs were treated with D-glucose $(0-50 \mathrm{mM})$, L-glucose $(25$ $\mathrm{mM})$, and D-mannitol $(25 \mathrm{mM})$ for 24 $\mathrm{h}$ and permeability was monitored by measuring the flux of Evans-blue-dyebound albumin across HUVECs. (B) HUVECs were treated with D-glucose $(25 \mathrm{mM})$ for indicated time periods and permeability was monitored. (C) The effects of pretreatment with different concentrations of baicalin (white bar), baicalein (gray bar) and wogonin (black bar) for $6 \mathrm{~h}$ on barrier disruptions caused by $25 \mathrm{mM} \mathrm{HG}$ for $24 \mathrm{~h}$. (D) The effects of baicalin (white bar), baicalein (gray bar) and wogonin (black bar) injected intravenously on HG-induced $(9 \mathrm{mg} /$ mouse, i.v.) vascular permeability in mice were determined by measuring the levels of Evans blue dye in peritoneal washings (expressed $\mu \mathrm{g} /$ mouse, $\mathrm{n}=5$ ). ( $\mathrm{E}$ ) The effects of baicalin (white bar), baicalein (gray bar) and wogonin (black bar) on cellular viability were measured using MTT assays. Results are expressed as the mean \pm SEM of at least three independent experiments. ${ }^{*} P<0.05$ versus $0(A, B), H G$ alone $(C, D)$. 
viability of baicalin, baicalein, or wogonin, MTT assays were performed on HUVECs treated with each compound for $24 \mathrm{~h}$. At the concentrations used (up to $50 \mu \mathrm{M}$ ), baicalin, baicalein, or wogonin did not affect the viability of HUVECs (Fig. 1E). These findings indicated the inhibitory effects of baicalin, baicalein, and wogonin on HG-mediated endothelial dysfunctions and barrier disruptive responses in mice. Therefore, prevention of HG-induced barrier disruption by each compound suggested the potential of baicalin, baicalein, or wogonin to treat vascular inflammatory diseases.

\section{Effects of polyphenols on HG-mediated expression of cell adhesion molecules (CAMs) and THP-1 adhesion}

Two important phenomena that occur early in the pathology of atherosclerosis are the adhesion of leukocytes to the endothelial layers, followed by the migration into the inflammatory sites, and enhanced vascular permeability $(15,17)$. Enhanced interactions between leukocyte and endothelial cells have been demonstrated in in vivo and in vitro diabetes models $(17,18)$. NF-אB activation is the major controller in enhanced expressions of CAMs and migration of leukocytes through endothelium by HG $(19,20)$. In addition, it is well known that the upregulation of CAMs is involved in the pathology of atherosclerosis (21). The interactions between endothelium and leukocytes such as the adhesion and migrations of leukocytes, which is a precursor to atheroma (21). Particularly, upreluation of CAMs such as vascular cell adhesion molecule-1 (VCAM-1), intracellular adhesion molecule-1 (ICAM-1), and E-selectin has been observed in the endothelial cells of human atherosclerotic lesions (22). The expression of CAMs on endothelial cells by $\mathrm{HG}$ has been widely studied. It was reported that HG increased the expression of ICAM-1 in human aortic endothelial cells (23); this study is consistent with findings indicating that $\mathrm{HG}$ is a mediator of leukocyte adhesion toward endothelial cells, counting on the enhanced expressions of VCAM-1, ICAM-1, and E-selectin (20). Furthermore, the adhesion of leukocytes toward the endothelial cells is followed by their migration and differentiation into macrophages, which is controlled by an interaction between the monocytes and CAMs (24). Therefore, we evaluated the effects of $\mathrm{HG}$ on the expression of CAMs and adhesion of monocytes to HUVECs in response to HG. The HG concentration-based responses in the expression of CAMs, such as VCAM-1, ICAM-1, and E-selectin, were determined by enzyme-linked immunosorbent assay. Exposure of the primary cultured HUVECs to HG resulted in significantly increased expression of CAMs after incubation with $25 \mathrm{mM}$ D-glucose; the maximum inhibitory effect of baicalin, baicalein, or wogonin (Fig. 2A) was observed at $10 \mu \mathrm{M}$.

And, to determine the effect of baicalin, baicalein, or wogonin on the interaction between endothelial cell and leukocyte, we tested the adhesion of THP-1 cells to HG-activated HUVECs and the migration of leukocytes in vivo. Adhesion of THP-1 cells to HUVECs was increased significantly with HG treatments. Pretreatment with baicalin, baicalein, or wogonin $(10 \mu \mathrm{M})$ resulted in a decreased number of THP-1 cells adhering to HG-induced HUVECs (Fig. 2B and 2C). These results were cor-
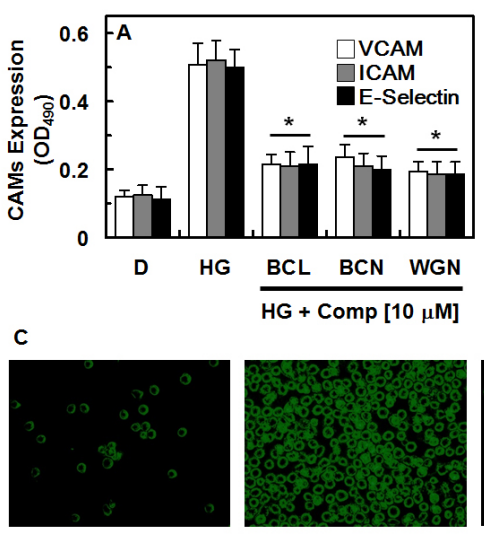

Cont

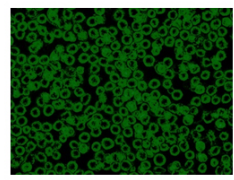

HG [25mM]

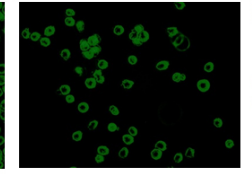

$H G+B C L[10 \mu M]$
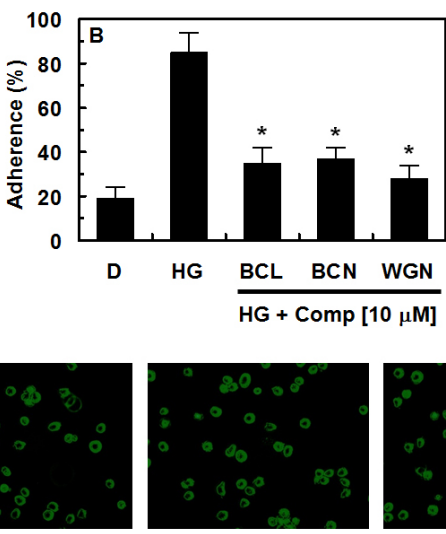

$H G+B C N[10 \mu M]$

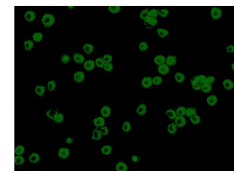

$H G+W G N[10 \mu M]$

$\stackrel{\square}{\circ}$

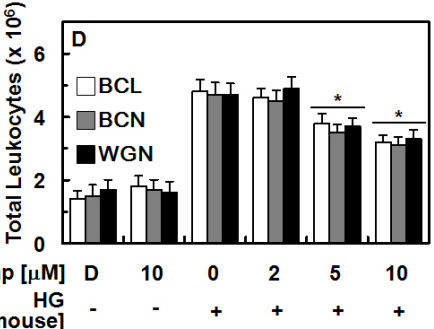

Fig. 2. Effects of polyphenols on HGmediated pro-inflammatory responses. HG-induced (25 mM, for $24 \mathrm{~h}$ ) expression of cell adhesion molecules on HUVECs was determined after treatment of cells with the indicated concentrations of baicalin, baicalein and wogonin for $6 \mathrm{~h}$. VCAM-1 (white bar), ICAM-1 (gray bar), and E-Selectin (black bar) were detected by ELISA. (B, C) HG-induced (25 mM, for 24 h)-mediated adherence of monocytes to HUVEC monolayers was assessed after pretreatment of cells with baicalin, baicalein and wogonin for $6 \mathrm{~h}$. The amounts of adherent THP-1 cells were monitored by (B) cell-cell adhesion assay and (C) fluorescence microscopy. (D) The same as Fig. 1D except that the leukocyte migration into the peritoneal cavities of mice was analyzed. Data are expressed as the mean \pm SEM of three independent experiments. ${ }^{* P}<0.05$ and ${ }^{*} \mathrm{P}<0.05$ vs. HG alone. 

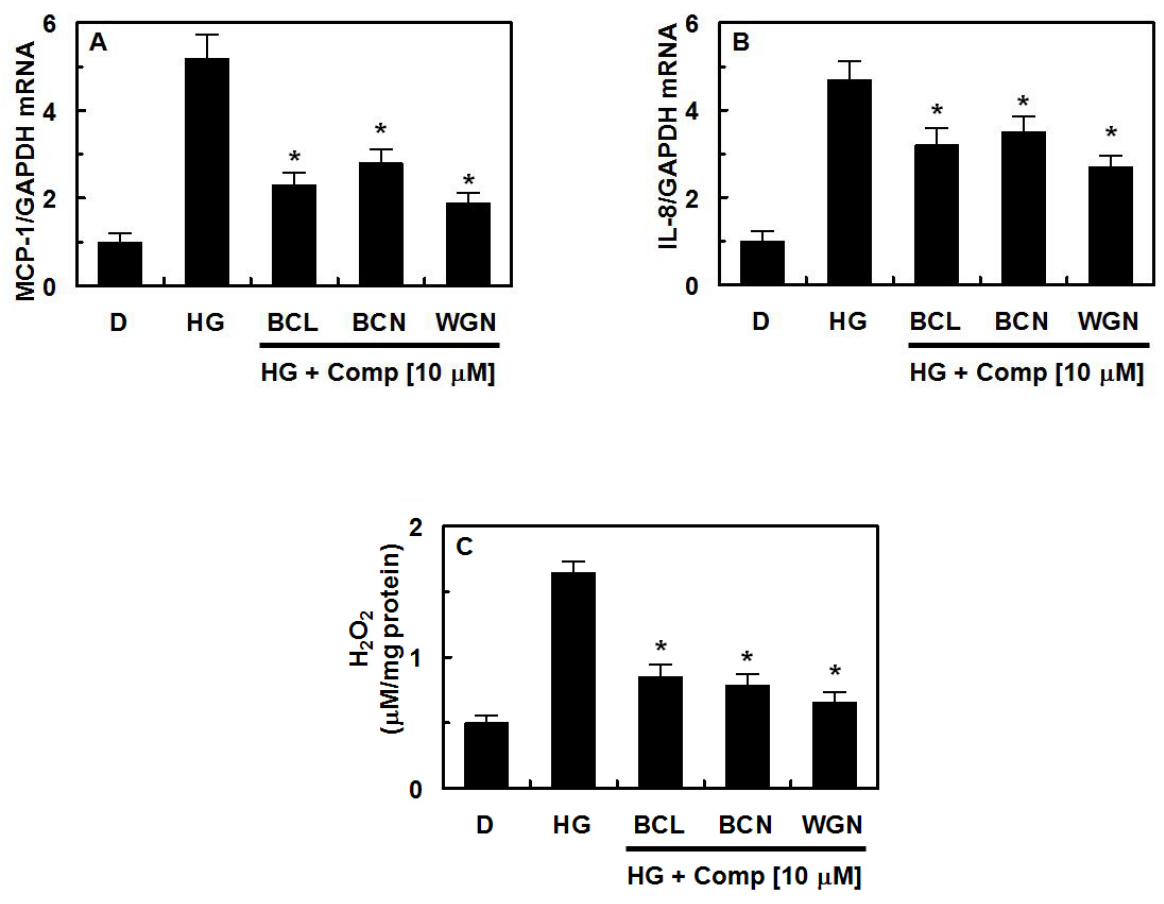

Fig. 3. Effects of polyphenols on HGinduced expression of MCP-1 and IL-8 mRNA and ROS formation. (A, B) Cells were pretreated with baicalin baicalein and wogonin for $6 \mathrm{~h}$ and then incubated with $\mathrm{HG}(25 \mathrm{mM})$ for 48 h. mRNA was extracted, and real time qRT-PCR analysis was performed using specific primer for MCP-1 (A), IL-8 (B), and GAPDH, as described in the Materials and methods section. (C) Cells were pretreated with baicalin, baicalein and wogonin for $6 \mathrm{~h}$ and then stimulated with $\mathrm{HG}$ for $1 \mathrm{~h}$; $\mathrm{H}_{2} \mathrm{O}_{2}$ assay was then performed as described in the Materials and methods section. Data are expressed as the mean \pm SEM of three independent experiments. ${ }^{*} \mathrm{P}<0.05$ vs. HG alone. roborated in vivo by the inhibition of HG-induced migration of leukocytes in the peritoneal space (Fig. 2D). Thus, baicalin, baicalein, or wogonin could be a therapeutic drug candidate for diabetic vascular inflammation by targeting CAMs expression in the prevention of atherosclerotic lesions.

IL-8 and MCP-1 are chemokines strongly implicated in the atherogenesis processes (25). IL-8 funtions as a chemotactic for neutrophils and MCP-1 is a important mediator of monocyte trafficking (25). Therefore, we tested the hypothesis that baicalin, baicalein, and/or wogonin would inhibit HG-induced MCP-1 and IL-8 mRNA levels using real time reverse transcription polymerase chain reaction. As shown in Fig. $3 \mathrm{~A}$ and $3 \mathrm{~B}, \mathrm{HG}$ induced an increase in the expression levels of MCP-1 (up to 5.2-fold) and IL-8 (up to 4.7-fold) mRNA; pretreatment with baicalin, baicalein, or wogonin resulted in decreased expression levels of HG-induced MCP-1 and IL-8 mRNA. These results suggested that baicalin, baicalein, or wogonin might be useful in preventing the diabetic inflammatory process.

\section{Effect of polyphenols on HG-induced oxidative stress}

The synthesis of reactive oxygen species (ROS) is physiologically related with inflammatory responses (26). Previous observations have indicated that $\mathrm{HG}$ raises the oxidant stress and the synthesis of free radicals in various types of cells; ROS are the key mediator of various oxidative events such as extracellular matrix deposition and cell proliferation $(27,28)$. Therefore, to determine the cyto-protective effect of baicalin, baicalein, or wogonin on HG-induced oxidative stress, HG-induced cellular $\mathrm{H}_{2} \mathrm{O}_{2}$ concentrations were measured. $\mathrm{H}_{2} \mathrm{O}_{2}$ levels were statistically increased after incubation for 10 min with 25 $\mathrm{mM}$ glucose; the maximum concentrations were observed after $1 \mathrm{~h}$ incubation (data not shown). Therefore, $1 \mathrm{~h}$ incubation condition was chosen to analyze cellular ROS in further experiments. As shown in Fig. 3C, pretreatment with $10 \mu \mathrm{M}$ baicalin, baicalein, or wogonin significantly inhibited HG-induced increase in $\mathrm{H}_{2} \mathrm{O}_{2}$ levels. In addition, baicalin, baicalein, or wogonin alone did not mediate oxidative stress (data not shown), which suggested the importance of HG-mediated oxidative stress on HUVECs in determining the characteristics of diabetic complications and vascular inflammation.

\section{Effect of polyphenols on HG-induced activation of NF- $\mathrm{KB}$}

Activation NF- $\mathrm{KB}$ affects the uprelations of CAMs and induces interconnected activations of other pro-inflammatory chemoattractants and cytokines, which could provide the biological relationship between endothelial cell dysfunction and cell redox states (29). In addition, ROS activates various transcription factors in cultured endothelial cells, including NF-kB (30). First, we measured $\mathrm{HG}$-induced translocation of NF- $\kappa \mathrm{B}$ from the cytosol into the nucleus. NF- $\kappa B$ p65 proteins are the active subunits of the NF-кB complex. Increased levels of p65 proteins in the nuclear extracts of HUVECs treated with HG were shown using western blotting analysis, and the cytosolic extracts exhibited an appreciable loss of p65 protein content (Fig. $4 \mathrm{~A})$. And, treatment with baicalin, baicalein, or wogonin resulted in the inhibition of HG-induced increases in p65 NF-кB 


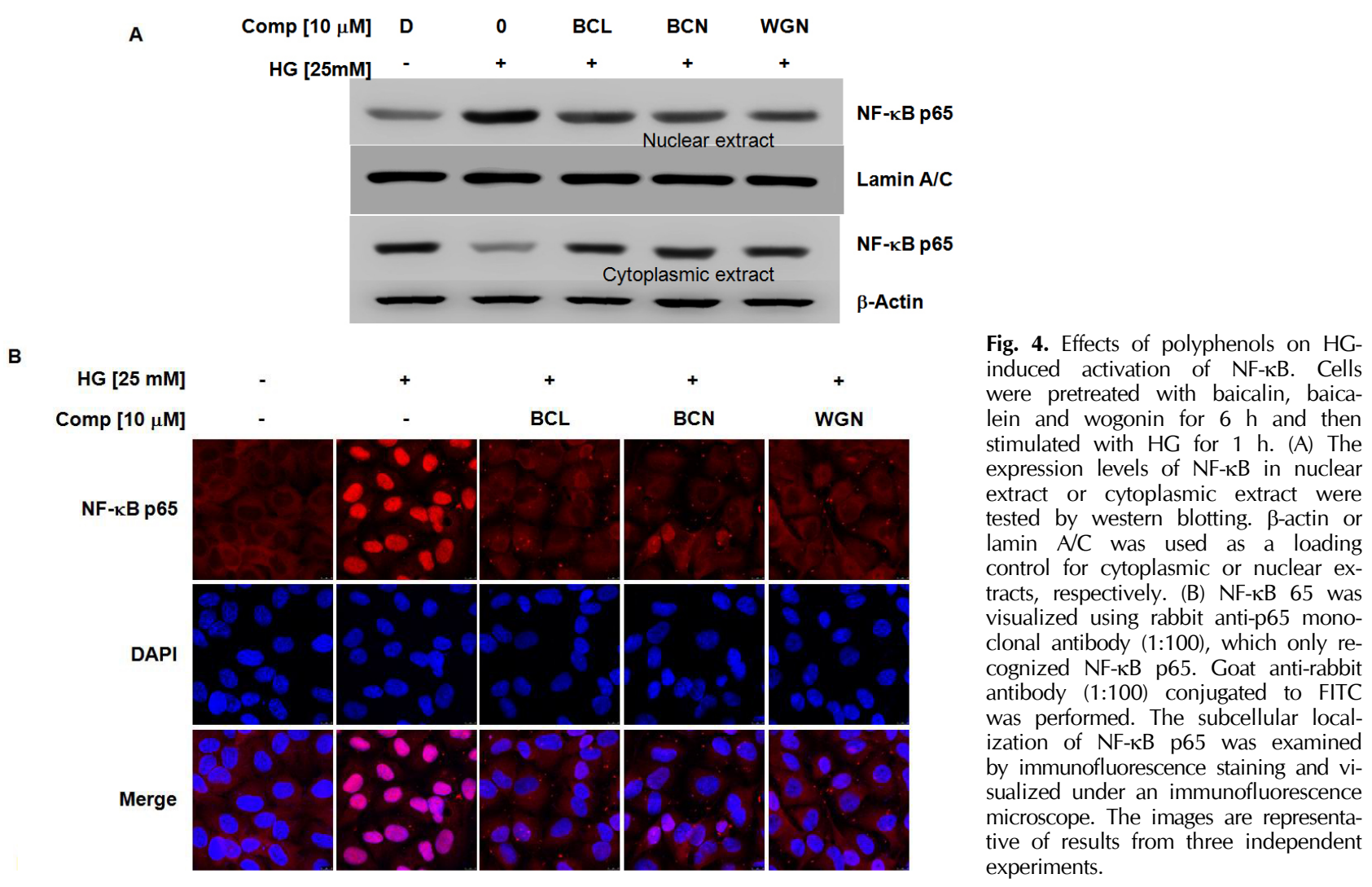

expression levels (Fig. 4A). To confirm the western blotting results, immunocytochemistry was applied using p65 NF- $\mathrm{kB}$ and FITC-conjugated antibodies. The results showed that HG increased the p65 NF- $\mathrm{KB}$ expression in the nucleus, whereas normal conditions did not. And, treatment with $10 \mu \mathrm{M}$ baicalin, baicalein, or wogonin resulted in a decrease in the HG-induced expression of p65 NF- $\mathrm{kB}$ in the nucleus, which were consistent with those of western blotting (Fig. 4A); they demonstrated that the HG-mediated NF- $\mathrm{KB}$ activation was inhibited by baicalin, baicalein, and wogonin, indicating that baicalin, baicalein, or wogonin have inhibitory effects on the $\mathrm{NF}-\kappa \mathrm{B}$ pathways specific to $\mathrm{HG}$-induced adhesion molecules on HUVECs.

In summary, our results demonstrated that treatment with baicalin, baicalein, or wogonin resulted in a blockage of HGinduced vascular inflammation due to the inhibitory effects of NF-KB in HUVECs. These results suggested that baicalin, baicalein, or wogonin have significant therapeutic benefits against diabetic complications and atherosclerosis by decreasing the $\mathrm{HG}$-induced generation of $\mathrm{H}_{2} \mathrm{O}_{2}$, increasing the activation of $\mathrm{NF}-\kappa \mathrm{B}$, expressions of CAMs, cell-cell adhesion/migration, and disruption of the endothelial barrier function. Our findings indicated that baicalin, baicalein, or wogonin are candidates for treating diabetic vascular inflammatory diseases.

\section{MATERIALS AND METHODS}

Please see the supplementary materials for materials and methods.

\section{ACKNOWLEDGEMENTS}

This study was supported by the National Research Foundation of Korea (NRF) funded by the Korea government [MSIP] (Grant Nos. 2012R1A5A2A42671316 and 2014R1A2A1A11049526).

\section{REFERENCES}

1. Whiting DR, Guariguata L, Weil C and Shaw J (2011) IDF diabetes atlas: global estimates of the prevalence of diabetes for 2011 and 2030. Diabetes Res Clin Pract 94, 311-321

2. Grundy SM, Benjamin IJ, Burke GL et al (1999) Diabetes and cardiovascular disease: a statement for healthcare professionals from the American Heart Association. Circulation $100,1134-1146$

3. Thomas JE and Foody JM (2007) The pathophysiology of cardiovascular disease in diabetes mellitus and the future of therapy. J Cardiometab Syndr 2, 108-113

4. Roglic G, Unwin N, Bennett PH et al (2005) The burden 
of mortality attributable to diabetes: realistic estimates for the year 2000. Diabetes Care 28, 2130-2135

5. Rubino F and Gagner M (2002) Potential of surgery for curing type 2 diabetes mellitus. Ann Surg 236, 554-559

6. Li GQ, Kam A, Wong KH et al (2012) Herbal medicines for the management of diabetes. Adv Exp Med Biol 771, 396-413

7. Day C (1998) Traditional plant treatments for diabetes mellitus: pharmaceutical foods. Br J Nutr 80, 5-6

8. Kubo M, Asano T, Shiomoto $\mathrm{H}$ and Matsuda $\mathrm{H}$ (1994) Studies on rehmanniae radix. I. Effect of $50 \%$ ethanolic extract from steamed and dried rehmanniae radix on hemorheology in arthritic and thrombosic rats. Biol Pharm Bull $17,1282-1286$

9. Kubo M, Matsuda H, Tanaka M et al (1984) Studies on Scutellariae radix. VII. Anti-arthritic and anti-inflammatory actions of methanolic extract and flavonoid components from Scutellariae radix. Chem Pharm Bull (Tokyo) 32, 2724-2729

10. Chen YC, Shen SC, Chen LG, Lee TJ and Yang LL (2001) Wogonin, baicalin, and baicalein inhibition of inducible nitric oxide synthase and cyclooxygenase-2 gene expressions induced by nitric oxide synthase inhibitors and lipopolysaccharide. Biochem Pharmacol 61, 1417-1427

11. Chiu JH, Lay IS, Su MY et al (2002) Tumor necrosis factor-producing activity of wogonin in RAW 264.7 murine macrophage cell line. Planta Med 68, 1036-1039

12. Laakso M (1999) Hyperglycemia and cardiovascular disease in type 2 diabetes. Diabetes 48, 937-942

13. Kannel WB and McGee DL (1979) Diabetes and cardiovascular disease. The Framingham study. JAMA 241, 2035-2038

14. Nannipieri M, Rizzo L, Rapuano A, Pilo A, Penno G and Navalesi R (1995) Increased transcapillary escape rate of albumin in microalbuminuric type II diabetic patients. Diabetes Care 18, 1-9

15. Wardle EN (1994) Vascular permeability in diabetics and implications for therapy. Diabetes Res Clin Pract 23, 135139

16. Tooke JE (1995) Microvascular function in human diabetes. A physiological perspective. Diabetes 44, 721-726

17. Gerrity RG (1981) The role of the monocyte in atherogenesis: I. Transition of blood-borne monocytes into foam cells in fatty lesions. Am J Pathol 103, 181-190

18. Esposito C, Fasoli G, Plati AR et al (2001) Long-term exposure to high glucose up-regulates VCAM-induced endo- thelial cell adhesiveness to PBMC. Kidney Int 59, 18421849

19. Hamuro M, Polan J, Natarajan M and Mohan S (2002) High glucose induced nuclear factor kappa B mediated inhibition of endothelial cell migration. Atherosclerosis 162, 277-287

20. Morigi M, Angioletti S, Imberti B et al (1998) Leukocyteendothelial interaction is augmented by high glucose concentrations and hyperglycemia in a NF-kB-dependent fashion. J Clin Invest 101, 1905-1915

21. Lopes-Virella MF and Virella G (1992) Immune mechanisms of atherosclerosis in diabetes mellitus. Diabetes 41 Suppl 2, 86-91

22. Bae JS (2012) Role of high mobility group box 1 in inflammatory disease: Focus on sepsis. Arch Pharm Res 35, 1511-1523

23. Kado S, Wakatsuki T, Yamamoto M and Nagata N (2001) Expression of intercellular adhesion molecule-1 induced by high glucose concentrations in human aortic endothelial cells. Life Sci 68, 727-737

24. Hansson GK and Libby $P$ (2006) The immune response in atherosclerosis: a double-edged sword. Nat Rev Immunol 6, 508-519

25. Boisvert WA (2004) Modulation of atherogenesis by chemokines. Trends Cardiovasc Med 14, 161-165

26. Inoguchi T, Li P, Umeda F et al (2000) High glucose level and free fatty acid stimulate reactive oxygen species production through protein kinase C--dependent activation of $\mathrm{NAD}(\mathrm{P}) \mathrm{H}$ oxidase in cultured vascular cells. Diabetes 49 , 1939-1945

27. Dunlop M (2000) Aldose reductase and the role of the polyol pathway in diabetic nephropathy. Kidney Int Suppl $77, \mathrm{~S} 3-12$

28. Han HJ, Lee YJ, Park SH, Lee JH and Taub M (2005) High glucose-induced oxidative stress inhibits $\mathrm{Na}$ //glucose COtransporter activity in renal proximal tubule cells. Am J Physiol Renal Physiol 288, F988-996

29. Rimbach G, Valacchi G, Canali R and Virgili F (2000) Macrophages stimulated with IFN-gamma activate NFkappa B and induce MCP-1 gene expression in primary human endothelial cells. Mol Cell Biol Res Commun 3, 238-242

30. Uemura S, Matsushita H, Li W et al (2001) Diabetes mellitus enhances vascular matrix metalloproteinase activity: role of oxidative stress. Circ Res 88, 1291-1298 